\title{
Membranas híbridas basadas en estireno-metacrilato-sílice y ácido fosfowolfrámico obtenidas por sol-gel para pilas de combustible de intercambio protónico (PEMFC)
}

\author{
J. MOSA, A. DURÁN, M. APARICIO \\ Instituto de Cerámica y Vidrio (CSIC). \\ Campus de Cantoblanco, Madrid, España
}

\begin{abstract}
Comparadas con los motores de combustión interna, las pilas de combustible de intercambio de protones (PEMFC) son capaces de operar sin emisiones de agentes contaminantes. El aumento de la temperatura de operación de la pila de combustible por encima de $100^{\circ} \mathrm{C}$ es uno de los grandes objetivos en este campo ya que facilitaría el desarrollo comercial de los vehículos eléctricos impulsados por pilas de combustible. Las membranas híbridas orgánico-inorgánicas nanoestructuradas combinan las propiedades necesarias para este tipo de aplicación. Se obtuvieron membranas híbridas dopadas con ácido fosfowolfrámico (PWA) por copolimerización radicálica a partir de alquilalcóxidos y monómeros de estireno y metacrilato, y por reacción sol-gel vía catálisis ácida. La conductividad protónica se logra realizando un proceso de sulfonación de anillos aromáticos para producir grupos $\mathrm{SO}_{3} \mathrm{H}$ unidos al anillo. Se ha estudiado el efecto de la sulfonación y el dopado de las membranas con PWA sobre las propiedades de la membrana, tales como la estabilidad química y térmica, retención de agua, capacidad de intercambio iónico y conductividad protónica. Las medidas de conductividad muestran aumento al aumentar la temperatura y la cantidad de grupos $\mathrm{SO}_{3} \mathrm{H}$, hasta valores de $3.210^{-3} \mathrm{~S} / \mathrm{cm}$ a $130^{\circ} \mathrm{C}$ y $100 \% \mathrm{HR}$.
\end{abstract}

Palabras clave: PEMFC; heteropoliácidos; sol-gel; membranas hibridas; sulfonación; conductividad protónica

Hybrid styrene-methacrylate-silica sol-gel membranes combining sulfonation and tungstophosphoric acid doping for PEMFC

Contrary to internal combustion engines, proton-exchange membrane fuel cells (PEMFC) used in transportation operate with zero emissions of environmental pollutants. The increase of the operation temperature in PEMFC above $100^{\circ} \mathrm{C}$ is a great concern for the application of this type of cells in electric vehicles. Hybrid organic-inorganic membranes with nanosized interfaces can combine the main properties of their components to meet this objective. Styrene-methacrylate-silica membranes doped with phosphotungstic acid (PWA) were prepared through acid catalyzed sol-gel process and free-radical copolymerization. Additionally, sulfonation processes of aromatic rings to produce attached $\mathrm{SO}_{3} \mathrm{H}$ groups were applied to increase the proton conductivity. The effect of sulfonation degree and PWA doping on the membrane properties such as chemical and thermal stability, water uptake, ion exchange capacity, and proton conductivity were investigated. The measurement of conductivity shows a general increase with rising temperatures and with the increasing of $\mathrm{SO}_{3} \mathrm{H}$ groups density, reaching a maximum value of $3.210^{-3} \mathrm{~S} / \mathrm{cm}$ at $130^{\circ} \mathrm{C}$ and $100 \% \mathrm{HR}$.

Keywords: PEMFC; heteropoly acids salts; sol-gel; hybrid membrane; sulfonation; proton conductivity

\section{INTRODUCCIÓN}

Las pilas de combustible de intercambio protónico (PEMFC) están recibiendo una especial atención durante estos últimos años debido a sus ventajas específicas frente a otros tipos de pilas de combustibles en aplicaciones en transporte, dispositivos electrónicos y otros $(1,2)$. Las pilas de combustible PEMFC requieren una membrana para separar tanto química como electrónicamente las reacciones químicas del ánodo y cátodo. Una membrana apropiada para una pila de combustible debe permitir el movimiento libre de los protones a través de ella. Hasta el momento se han aplicado casi exclusivamente como electrolito en PEMFC los polímeros perfluorados, principalmente el Nafion (Du Pont $\left.{ }^{\circledR}\right)$. Sin embargo, las membranas tipo Nafion tienen un intervalo de aplicación muy limitado, con una temperatura de uso por debajo de los $80^{\circ} \mathrm{C}$ y necesitan una humidificación del $100 \%$. Por tanto, una de las áreas de mayor interés en estas pilas es el desarrollo de membranas de bajo coste para aplicaciones de alta temperatura $\left(\mathrm{T}>100^{\circ} \mathrm{C}\right)$ usando hidrógeno o metanol (DMFC) como combustible. Operar a alta temperatura tiene grandes ventajas como la mejora de las cinéticas de reacción de los electrodos, menor consumo de metal catalítico $(\mathrm{Pt})$ y reducción del envenenamiento del platino con trazas de $\mathrm{CO}$ $(3,5)$.

Las membranas híbridas nanoestructuradas son una alternativa a las membranas poliméricas para alcanzar altas temperaturas de operación en la pila. En estas membranas, el componente inorgánico proporciona la estabilidad térmica, mientras que propiedades como flexibilidad o resistencia a la manipulación están ligadas al componente orgánico. Asimismo, la conductividad protónica se relaciona con la red inorgánica debido a la capacidad de retención de moléculas de agua en los grupos hidroxilo superficiales (6-11). Un 
modo de mejorar la conductividad protónica es dopar las membranas híbridas con heteropoliácidos (HPAs) como el

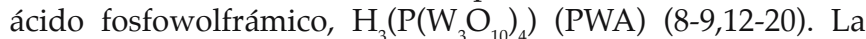
utilización de heteropoliácidos como dopantes en membranas para pilas de combustible permite alcanzar elevados valores de conductividad, aunque se han apreciado problemas de lixiviación de los HPA en el agua generada en el cátodo (12).

Una de las formas de solucionar este problema sería retener el PWA dentro de la membrana, uniéndolo a la red a través de enlaces químicos. Los agregados aniónicos de PWA se pueden estabilizar dentro de la membrana a través de interacciones fuertes de tipo iónico con la red inorgánica. Si además el PWA retenido en la red está homogéneamente distribuido y en estado nanométrico, la fase fuertemente ácida e hidrofílica origina un camino de conducción de los protones a través de diferentes canales (12). Otra manera de incrementar la conductividad protónica es el anclaje de grupos $\mathrm{SO}_{3} \mathrm{H}$ a través de la reacción de sulfonación de anillos aromáticos (21-22).

En trabajos previos $(9,23)$ se estudió el sistema TEOSHEMA-STY dopado con PWA alcanzándose elevados valores de conductividad protónica, pero las membranas mostraban baja estabilidad química en medio acuoso. Este trabajo tiene como objetivo mejorar la estabilidad mecánica y química de las membranas variando la composición. Para ello se ha sustituido el tetraetil ortosilicato (TEOS) por el 3-metacriloxipropil trimetoxisilano (MPS), precursor híbrido, que actúa de nexo de unión entre la red orgánica e inorgánica, aumentando el entrecruzamiento y, mejorando la estabilidad química de la membrana. No obstante, el entrecruzamiento y la densidad de la membrana deben controlarse para mantener abiertos los canales protónicos de conducción y no disminuir la conductividad protónica. Por otro lado, el MPS que polimeriza formando cadenas largas, incrementará la flexibilidad y la estabilidad mecánica de la membrana. Uno de los objetivos de este trabajo ha sido el control de las condiciones de síntesis para producir de forma simultánea la copolimerización orgánica radicálica del MPS con los monómeros 2-hidroxietil metacrilato (HEMA) y estireno (STY), y la inorgánica por reacciones de hidrólisis y policondensación a través de los enlaces hidrolizables del MPS. La incorporación del STY, que posee anillos aromáticos susceptibles de sulfonarse, permite combinar los dos métodos descritos para obtener conductividad protónica: el proceso de sulfonación y la incorporación del PWA como dopante.

\section{PARTE EXPERIMENTAL}

\subsection{Síntesis}

Se usaron como precursores 3-metacriloxipropil trimetoxisilano (MPS) de ABCR, estireno (STY) de Aldrich, ácido fosfowolfrámico hidratado (PWA) y 2- hidroxietil metacrilato (HEMA) de Fluka. El sol, de composición molar 40 HEMA - 19.3 MPS - 40 STY - 0.7 PWA (HMS-PWA) se obtuvo a partir de la mezcla de dos soluciones. La solución A se preparó mezclando etanol absoluto (relación molar etanol/MPS = 2.6), con PWA y $\mathrm{H}_{2} \mathrm{O}$ (en relación molar agua/MPS $=2$ ), agitando a temperatura ambiente durante 30 minutos. La solución B contiene la mezcla de HEMA, STY y el iniciador de la reacción de polimerización radicálica 2,2'-azobis(isobutironitrilo) (AIBN, de FLUKA) (0.85 p-p\% respecto a la suma de MPS+HEMA+STY). El sol se agita a temperatura ambiente durante 30 minutos y posteriormente se añade el MPS, manteniendo la agitación otros 15 minutos. A continuación se mezclan ambas soluciones y se continúa agitando 15 minutos. El PWA actúa como catalizador ácido. Los soles se cuelan en moldes de politetrafluoroetileno (PTFE) y de vidrio, y se tratan a $50^{\circ} \mathrm{C}$. Las membranas obtenidas se trataron adicionalmente a $150^{\circ} \mathrm{C}$ (velocidad de calentamiento $0.1^{\circ} \mathrm{C} / \mathrm{min}$ ) en aire durante 24 horas para completar el curado. Las membranas se sulfonaron por inmersión durante tres horas en soluciones $0.3 \mathrm{M}$ de ácido clorosulfónico $\left(\mathrm{HSO}_{3} \mathrm{Cl}\right)$ y de ácido trimetilsililclorosulfonato $\left(\left(\mathrm{CH}_{3}\right)_{3} \mathrm{SiSO}_{3} \mathrm{Cl}\right)$ (de Panreac y Aldrich, respectivamente) en 1,2-dicloroetano.

\subsection{Caracterización}

\subsubsection{Microscopia electrónica de barrido}

La superficie de fractura de las membranas se examinó con un microscopio electrónico de barrido de emisión de campo (FE-SEM) HITACHI S-4700. La distribución de los elementos a través del espesor de la membrana se analizó por EDX (NORAN system six).

\subsubsection{Análisis térmico (ATG-ATD) y estructural (FTIR)}

El comportamiento térmico (ATG-ATD) de las muestras tratadas a $150^{\circ} \mathrm{C}$ se realizó usando un equipo Netzsch STA 409 con una velocidad de calentamiento de $10^{\circ} \mathrm{C} / \mathrm{min}$ en aire hasta $900^{\circ} \mathrm{C}$. Los espectros de FTIR se obtuvieron utilizando un espectrofotómetro Brucker en el intervalo de 4000-400 cm 1 .

\subsubsection{Absorción de agua}

La capacidad de absorción de agua de la membrana híbrida se determina a partir del cambio de peso después de sumergir la membrana en agua desionizada durante 24 horas. Tras la eliminación del agua superficial se determina el peso de la membrana húmeda. El peso de la membrana seca se mide después del secado a $150^{\circ} \mathrm{C}$ durante 24 horas. La capacidad de absorción de agua se calcula a través de la siguiente formula:

Absorción $\mathrm{H}_{2} \mathrm{O}(\%)=[($ Peso húmedo $(\mathrm{g})$ - Peso seco $(\mathrm{g})) /$ Peso $\operatorname{seco}(g)] 100$

\subsubsection{Capacidad de intercambio iónico}

Las membranas de intercambio protónico se caracterizan por una capacidad de intercambio iónico (IEC/ $\mathrm{mmol} \bullet \mathrm{g}^{-1}$ ) definible como el número de milimoles de $\mathrm{H}^{+}\left(\mathrm{n}^{+}\right)$por unidad de masa de la membrana seca $\left(\mathrm{m}_{\text {seco }}\right)$ :

$$
\mathrm{IEC}=\mathrm{n} \mathrm{H}^{+} / \mathrm{m}_{\text {seco }}
$$

Las membranas se protonan por inmersión en una solución de $\mathrm{HCl} 1 \mathrm{~N}$ durante 24 horas. Posteriormente se sumergen en $50 \mathrm{ml}$ de $\mathrm{NaCl} 1 \mathrm{M}$ durante otras 24 horas para producir el intercambio iónico entre los protones de la membrana y los iones sodio. La solución se valora con $\mathrm{NaOH} 0.005 \mathrm{M}$ hasta punto de equivalencia y las membranas se protonan con $\mathrm{HCl}$ $1 \mathrm{~N}$, eliminándose el exceso de agua e introduciéndose en una estufa de vacío a $80^{\circ} \mathrm{C}$ hasta obtener un peso constante $\left(\mathrm{m}_{\text {seco }}\right)$.

\subsubsection{Conductividad protónica}

La conductividad protónica de las membranas híbridas tratadas a $150^{\circ} \mathrm{C}$ se mide por Espectroscopia de Impedancia Compleja (EIS) usando un equipo Gamry FAS2 Femtostat, medida en dos puntas con eléctrodos de plata en la dirección transversal de la muestra y un intervalo de frecuencias de 1 
a $10^{5} \mathrm{~Hz}$. Las medidas se realizan en función de la humedad relativa y de la temperatura. Las muestras se estabilizan durante 24 horas a la humedad relativa deseada dentro de la celda de conductividad, que es un compartimento estanco que contiene una sal saturada de $\mathrm{NaNO}_{3}$ que proporciona una humedad relativa del $62-70 \%$ HR entre temperatura ambiente y $50^{\circ} \mathrm{C}, 70-81 \% \mathrm{HR}$ entre $50^{\circ} \mathrm{C}$ y $70^{\circ} \mathrm{C}, 81-99 \%$ HR entre $70^{\circ} \mathrm{C}$ y $90^{\circ} \mathrm{C}$, y $100 \% \mathrm{HR}$ a partir de $90^{\circ} \mathrm{C}$. La temperatura y humedad relativa de la celda se controla a través de una sonda Rotronic HYGROCLIP HK 25.

\section{RESULTADOS Y DISCUSIÓN}

\subsection{Homogeneidad y estabilidad mecánica}

Las membranas híbridas tratadas a $50^{\circ} \mathrm{C}$ resultaron homogéneas, transparentes y de color amarillo. El tratamiento térmico hasta $150^{\circ} \mathrm{C}$ cambia el color de las membranas a marrón oscuro con una pérdida parcial de transparencia, aunque sin aparición de grietas. El proceso de sulfonación con soluciones de $\mathrm{HSO}_{3} \mathrm{Cl}$ y $\left(\mathrm{CH}_{3}\right)_{3} \mathrm{SiSO}_{3} \mathrm{Cl}$ no produce cambios apreciables en las membranas, obteniéndose muestras de gran tamaño (5 $\mathrm{cm}^{2}$ ) con alta estabilidad mecánica, tal como se observa en la fotografía de la membrana sulfonada con solución de $\mathrm{HSO}_{3} \mathrm{Cl}$ obtenida en molde de teflón (Figura 1).

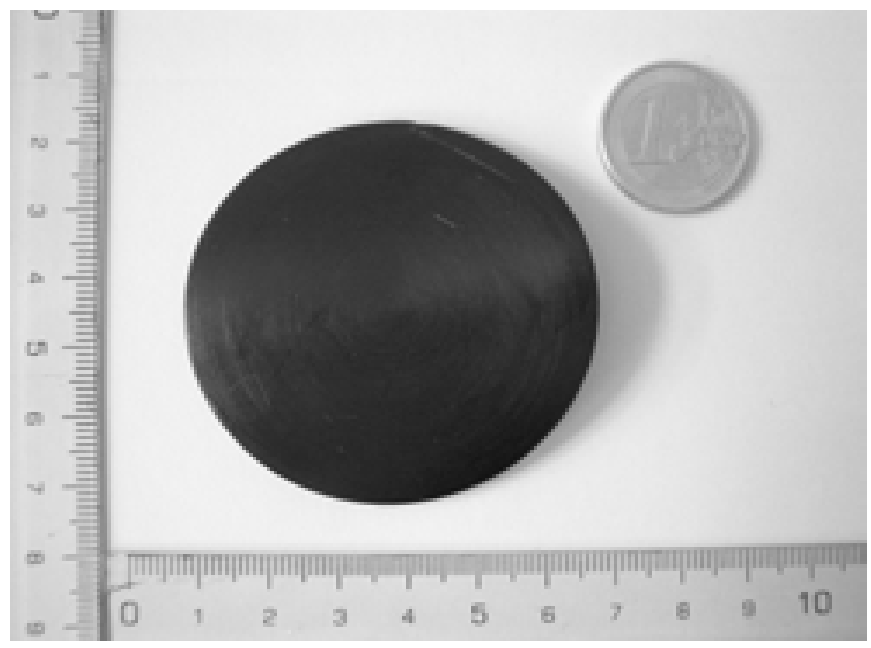

Fig. 1- Fotografía de la membrana de composición HMS-PWA sulfonada con solución de $\mathrm{HSO}_{3} \mathrm{Cl}$ y tratada a $150^{\circ} \mathrm{C}$.

\subsection{Aspectos estructurales}

La superficie de fractura de la membrana HMS-PWA sulfonada con solución de $\left(\mathrm{CH}_{3}\right)_{3} \mathrm{SiSO}_{3} \mathrm{Cl}$ y tratada a $150^{\circ} \mathrm{C}$ fue analizada por MEB (emisión de campo). La fotografía (Figura 2) muestra un material homogéneo formado por nano-aglomerados ( $50 \mathrm{~nm})$ sin separación de fases, con una porosidad muy pequeña $(\sim 10 \mathrm{~nm})$, importante para retener el agua a alta temperatura. Los análisis de EDX confirman una distribución homogénea de elementos y en particular del PWA.

La Figura 3 presenta el espectro de transmisión FTIRATR de las membranas HMS dopadas con PWA junto con las dopadas y sulfonadas con ambos agentes sulfonantes $\mathrm{HSO}_{3} \mathrm{Cl}$ y $\left(\mathrm{CH}_{3}\right)_{3} \mathrm{SiSO}_{3} \mathrm{Cl}$. Las asignaciones de las bandas principales (Tabla I) están basadas en los valores de la literatura $(8,21,23-$ 24). Las bandas presentes en el espectro del PWA concuerdan con todas las bandas asignadas en la literatura a los iones

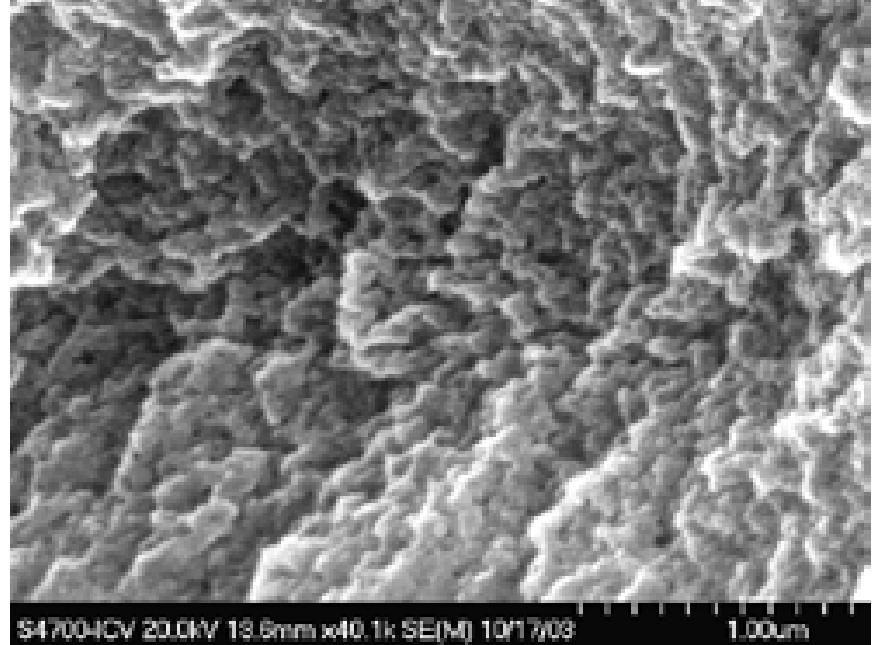

Fig. 2- Fotografía MEB (emisión de campo) de la superficie de fractura de la membrana de composición HMS-PWA sulfonada con solución de $\left(\mathrm{CH}_{3}\right)_{3} \mathrm{SiSO}_{3} \mathrm{Cl}$ y tratada a $150^{\circ} \mathrm{C}$.

Keggin, indicando que la geometría de estos iones se mantiene dentro de la estructura híbrida. La banda estrecha a $800 \mathrm{~cm}^{-1}$, asignada a $\mathrm{W}-\mathrm{O}_{\mathrm{c}}-\mathrm{W}$ en el PWA puro, se desplaza a $810 \mathrm{~cm}^{-1}$ cuando el PWA se incorpora a la estructura de la membrana. Este comportamiento se puede explicar por una adecuada separación entre las unidades Keggin y una vibración menos distorsionada por las interacciones anión-anión (24). Además, se observa un ligero desplazamiento de la banda $\mathrm{W}-\mathrm{O}_{\mathrm{b}}-\mathrm{W}$ a $885 \mathrm{~cm}^{-1}$ del espectro del PWA puro hasta $890 \mathrm{~cm}^{-1}$ en las membranas híbridas dopadas con PWA. Estos cambios en las frecuencias podrían indicar una interacción entre los grupos hidroxilo del HEMA y de los silanoles del MPS con las unidades Keggin del PWA (13), que estabilizaría el PWA en la membrana $(13,18)$. Se observa además un pequeño pico a 1636 $\mathrm{cm}^{-1}$ asignado a los enlaces $\mathrm{C}=\mathrm{C}$ remanentes, cuya oxidación probablemente origine el cambio de color observado en las membranas después del tratamiento térmico a $150^{\circ} \mathrm{C}$. El proceso de sulfonación parece no afectar a las bandas asignadas a los componentes metacrilato, estireno y PWA, indicando que la estructura es estable a este proceso.

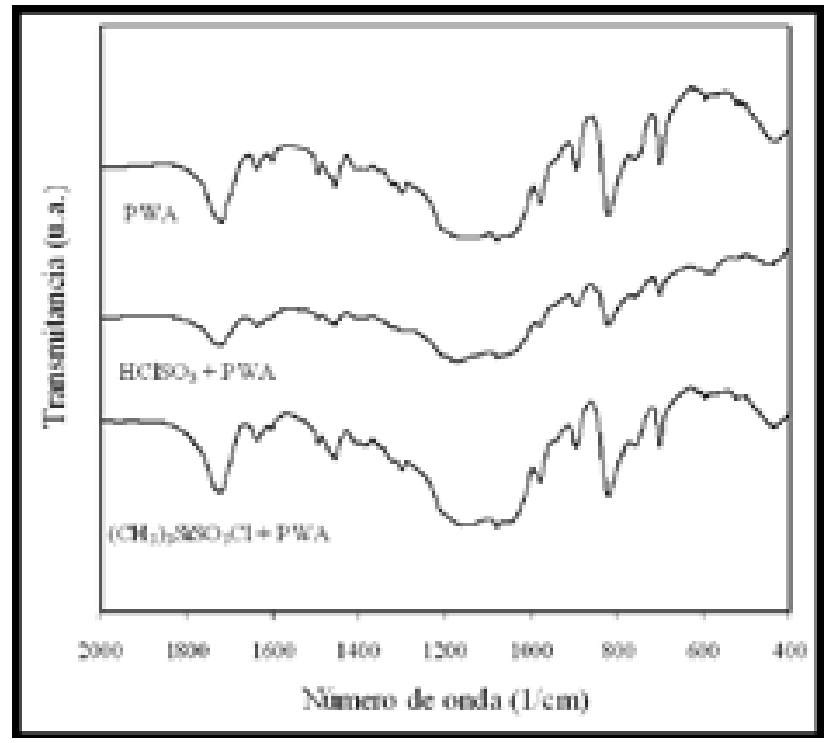

Fig. 3- Espectros de transmisión FTIR de las membranas HMS-PWA sin sulfonar y sulfonadas con soluciones de $\mathrm{HSO}_{3} \mathrm{Cl}$ y $\left(\mathrm{CH}_{3}\right)_{3} \mathrm{SiSO}_{3} \mathrm{Cl}$. 
TABLA I. ASIGNACIONES DE LAS PRINCIPALES BANDAS DE ABSORCIÓN

\begin{tabular}{|c|c|}
\hline Numero de Onda $\left(\mathrm{cm}^{-1}\right)$ & Enlace asignado \\
\hline 530,581 & PWA \\
\hline 810 & $\mathrm{~W}-\mathrm{O}_{\mathrm{c}}-\mathrm{W}$ \\
\hline 890 & $\mathrm{~W}-\mathrm{O}_{\mathrm{b}}-\mathrm{W}$ \\
\hline 980 & $\mathrm{~W}-\mathrm{O}_{\mathrm{d}^{\prime}} \mathrm{Si}-\mathrm{OH}$ \\
\hline 1090 & Si-O-Si, C-O-C, P-O \\
\hline 695 & O-Si-O, Si-C \\
\hline 1023,1200 & $\mathrm{SO} 3, \mathrm{Si}-\mathrm{O}-\mathrm{Si}$ \\
\hline 920 & SO \\
\hline $700,745,1375,1450,1590$ & fenilos \\
\hline $1170,1300-1600$ & metacrilato \\
\hline 1630 & $\mathrm{C}=\mathrm{C}$ \\
\hline 1720 & $\mathrm{C}=\mathrm{O}$ \\
\hline
\end{tabular}

\subsection{Estabilidad térmica}

Las curvas ATG-ATD de las membranas dopadas con PWA y, dopadas y sulfonadas con una solución de $\left(\mathrm{CH}_{3}\right)_{3} \mathrm{SiSO}_{3} \mathrm{Cl}$, aparecen en la Figura 4. Las curvas termogravimétricas muestran tres regiones: la primera, comprendida entre temperatura ambiente y $250^{\circ} \mathrm{C}$, muestra un pico endotérmico pequeño y ancho centrado a $240^{\circ} \mathrm{C}$ y una ligera pérdida de peso atribuida a la desorción física del agua absorbida, a los solventes residuales y al agua estructural del PWA (18). La pérdida de peso es relativamente baja debido a que las membranas han sido tratadas a $150^{\circ} \mathrm{C}$. La segunda región, comprendida entre 250 y $450^{\circ} \mathrm{C}$, presenta una importante pérdida de peso y un pico exotérmico a $370^{\circ} \mathrm{C}$, asignado a la combustión parcial de los componentes orgánicos, principalmente grupos metacrilato provenientes del HEMA y MPS, y a la descomposición de los grupos $\mathrm{SO}_{3}^{-}$. En esta región se observan las principales diferencias entre las membranas con y sin sulfonación. Las membranas no sulfonadas presentan una pérdida de peso del $28 \%$, mientras las sulfonadas presentan la pérdida de peso del $42 \%$ debido a la eliminación adicional de los grupos $\mathrm{SO}_{3}$. Por encima de $450^{\circ} \mathrm{C}$ aparece un

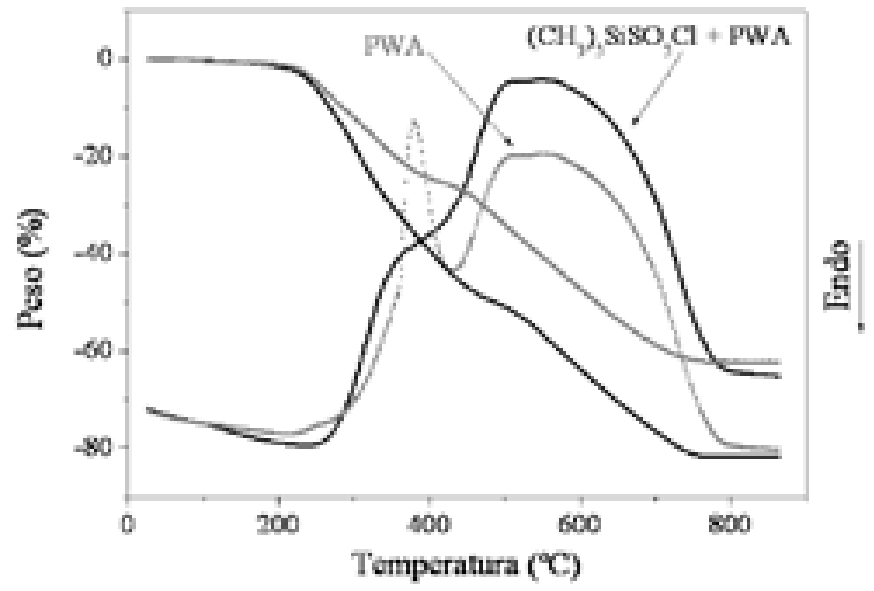

Fig. 4- Curvas ATG-ATD de las membranas HMS-PWA y, HMS-PWA sulfonada con $\left(\mathrm{CH}_{3}\right)_{3} \mathrm{SiSO}_{3} \mathrm{Cl}$. ancho pico exotérmico entre 470 y $750^{\circ} \mathrm{C}$, y una pérdida de peso continua hasta $800^{\circ} \mathrm{C}$. Ambos procesos pueden asignarse a la descomposición final de los grupos orgánicos, sobre todo anillos aromáticos y al colapso de la estructura del PWA. Tanto la pequeña pérdida de peso observada hasta $\operatorname{los} 200^{\circ} \mathrm{C}$, como la posición del pico exotérmico de menor temperatura (que comienza a $250^{\circ} \mathrm{C}$ ), indican que la estabilidad térmica de estas membranas híbridas es suficiente para su uso en pilas de combustible de intercambio protónico a temperaturas de al menos $150^{\circ} \mathrm{C}$.

3.4. Absorción de agua y capacidad de intercambio iónico

La capacidad de retención de agua de la membrana se determinó por variación del peso antes y después de la hidratación. Las membranas híbridas presentan absorción de agua tras el tratamiento de consolidación de la estructura a $150^{\circ} \mathrm{C}$. La membrana dopada con PWA incrementa su peso por hidratación en un $7.6 \%$, debido a las propiedades hidrofílicas del PWA y al MPS, que genera una red inorgánica que proporciona grupos hidroxilo y porosidad de tamaño nanométrico que permite retener las moléculas de agua. Las membranas sulfonadas con soluciones de $\mathrm{HSO}_{3} \mathrm{Cl}$ y $\left(\mathrm{CH}_{3}\right)_{3} \mathrm{SiSO}_{3} \mathrm{Cl}$ presentan absorciones mayores entre $10.3 \%$ y $8.9 \%$ respectivamente, debido a que los grupos sulfonato son hidrofilícos y absorben agua. El proceso de sulfonación con $\mathrm{HSO}_{3} \mathrm{Cl}$ es más efectivo, generando mayor número de grupos sulfonato, y por lo tanto una mayor absorción de agua. Los valores de retención de agua son relativamente altos y adecuados para la aplicación de estas membranas en PEMFC, ya que son materiales hidrofílicos.

La capacidad de intercambio iónico es indicativa de la proporción de los protones intercambiables en la membrana, que son los responsables de la conducción protónica. El IEC de la membrana de composición HMS-PWA sin sulfonar es muy bajo $\left(0.105 \mathrm{mmol}^{\circ} \mathrm{g}^{-1}\right)$, debido a la baja concentración de PWA en la membrana, con este dopante como única fuente de protones. El IEC de las membranas que combinan el dopado con la sulfonación aumenta con la efectividad del grupo sulfonante, $0.43 \mathrm{mmol} \bullet \mathrm{g}^{-1}$ para $\mathrm{HSO}_{3} \mathrm{Cl}$ y $0.21 \mathrm{mmol} \bullet \mathrm{g}^{-1}$ para $\left(\mathrm{CH}_{3}\right)_{3} \mathrm{SiSO}_{3} \mathrm{Cl}$, al igual que la absorción de agua. La membrana dopada y sulfonada con $\mathrm{HSO}_{3} \mathrm{Cl}$ presenta la mayor densidad de posiciones iónicas ( $\mathrm{PWA}, \mathrm{SO}_{3}^{-}$), y por lo tanto el máximo valor de EIC. Sin embargo, este valor es significativamente menor que el del Nafion 115, situado en $0.91 \mathrm{mmol} \bullet \mathrm{g}^{-1}(25)$. Un valor menor de EIC implica menores valores de conductividad. Sin embargo, estas membranas muestran un menor aumento de volumen con la absorción de agua, lo que origina un mejor contacto membrana-electrodos, que contribuye a una posible mejora del comportamiento en la celda.

\subsection{Conductividad protónica}

Todos los espectros de impedancia compleja (diagrama Nyquist) presentan un semicírculo que pasa por el origen a altas frecuencias y una recta inclinada a bajas frecuencias. La Figura 5 muestra los diagramas Nyquist a diferentes temperaturas de la membrana sulfonada con $\mathrm{HSO}_{3} \mathrm{Cl}$. El semicírculo se explica con el típico circuito equivalente con una resistencia y una capacitancia conectadas en paralelo, y la recta índica la presencia de una impedancia de Warburg originada por la difusión de los protones a través de la membrana. La conductividad , $\sigma$, a través de la membrana se calcula usando la relación: $\sigma=\mathrm{e} / \mathrm{RA}$, donde e y A son el espesor de la membrana y el área del electrodo, y $\mathrm{R}$ es 


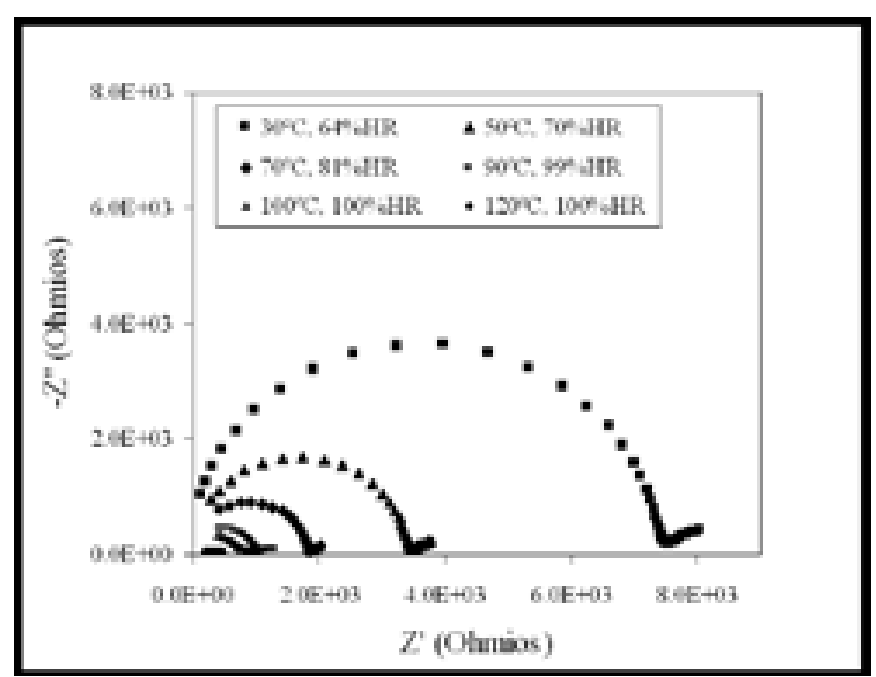

Fig. 5- Diagramas de Nyquist a diferentes temperaturas y humedades relativas para una membrana HMS-PWA sulfonada con $\mathrm{HSO}_{3} \mathrm{Cl}$.

la resistencia que se obtiene a través del punto de corte del semicírculo con el eje real de impedancias, Re ( $\left.Z^{\prime}\right)$, usando el programa de ajuste Echem Analyst de Gamry. La presencia de una sola constante de tiempo indica un único mecanismo de conducción, donde las moléculas de agua son el vehículo que permite el movimiento de los protones a través de la membrana. El proceso de sulfonación incorpora grupos $\mathrm{SO}_{3}$ que proporcionan un anclaje adicional a las moléculas de agua, y por tanto, nuevos puntos de apoyo para los protones, reduciendo la distancia entre ellos y explicando la mayor

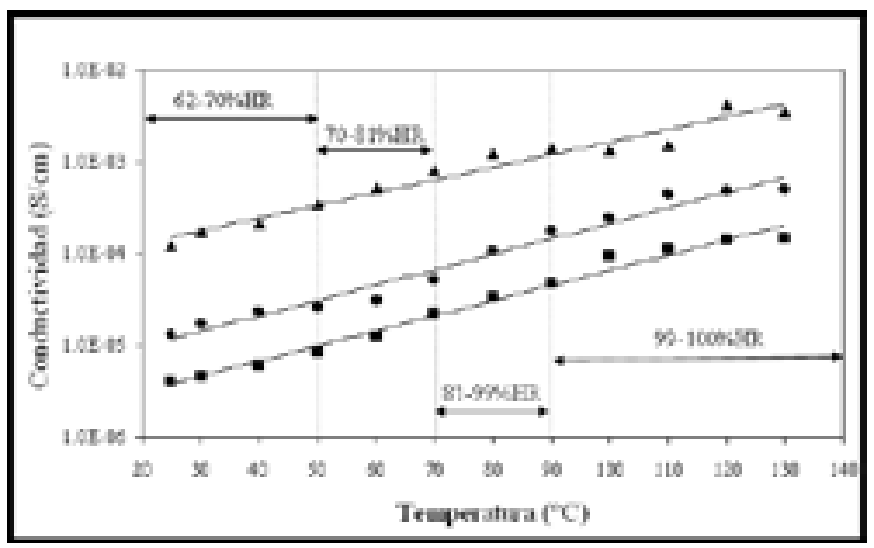

Fig. 6- Representación de la conductividad frente a la temperatura y humedad relativa para las composiciones HMS-PWA ( $(\mathbf{\square})$ y, HMS-PWA sulfonadas con soluciones de $\mathrm{HSO}_{3} \mathrm{Cl}(\boldsymbol{\Delta})$ y $\left(\mathrm{CH}_{3}\right)_{3} \mathrm{SiSO}_{3} \mathrm{Cl}(\bullet)$.

conductividad cuando se combinan los procesos de dopado con PWA y la sulfonación de anillos aromáticos.

La Figura 6 presenta los valores de conductividad de las membranas en función de la temperatura y la humedad relativa. La conductividad aumenta con la humedad relativa y la temperatura, indicando que el proceso de conducción protónica está activado térmicamente. La humedad relativa influye en la cantidad de moléculas de agua que ocupan posiciones reticulares en la estructura del PWA, y también en la cantidad de agua absorbida por la red de sílice y los grupos hidroxilo del HEMA, permitiendo el transporte de los protones a través del material. Las membranas sulfonadas presentan mayor conductividad que las sólo dopadas con PWA, debido a la presencia de los grupos $\mathrm{SO}_{3}$. Las membranas sulfonadas con $\mathrm{HSO}_{3} \mathrm{Cl}$ muestran conductividades un orden de magnitud mayor que las sulfonadas con $\left(\mathrm{CH}_{3}\right)_{3} \mathrm{SiSO}_{3} \mathrm{Cl}, 3.210^{-3}$ y 5.1 $10^{-4} \mathrm{~S} / \mathrm{cm}$ a $130^{\circ} \mathrm{C}$ y $100 \% \mathrm{HR}$ respectivamente, demostrando que el proceso de sulfonación con $\mathrm{HSO}_{3} \mathrm{Cl}$ es un método más eficiente para unir los grupos $\mathrm{SO}_{3}^{-}$a los anillos aromáticos debido a su mayor reactividad.

La conductividad protónica alcanza valores máximos de $3 \cdot 10^{-3} \mathrm{~S} / \mathrm{cm}$, para la membrana HMS-PWA sulfonada con $\mathrm{HSO}_{3} \mathrm{Cl}$, valor inferior en más de un orden de magnitud al obtenido con Nafion (25).

El objetivo de este trabajo ha sido mejorar las propiedades de estabilidad química, mecánica y térmica de la membrana del sistema HMS-PWA intentando mantener la conductividad protónica. Para ello buscamos el compromiso entre estas propiedades, optimizando la relación entre componente orgánico e inorgánico en el sistema HMS-PWA. Si comparamos los valores de conductividad protónica de este sistema, con los obtenidos en trabajos previos $(9,23)$, éstos son inferiores a pesar de la combinación del dopado con PWA y la sulfonación.

Para el sistema HEMA-STY-TEOS-PWA (23), la conductividad aumenta con la cantidad de sílice y PWA incorporados, alcanzándose valores similares al Nafion. Estos elevados valores son debidos a la gran absorción y retención de agua por parte de la sílice, y a la presencia del PWA, aunque presentan baja estabilidad química y mecánica. Sin embargo para el sistema HEMA-MPS-PWA (9) se alcanzan valores de conductividad menores que en el caso anterior, aunque un orden de magnitud superior $\left(3 \cdot 10^{-2} \mathrm{~S} / \mathrm{cm}\right.$ para membranas dopadas con $1.5 \%$ Molar de PWA) a los presentados en este trabajo, y también, baja estabilidad química y térmica. Aunque la conductividad protónica para el sistema HMS-PWA ha resultado menor que en los trabajos precedentes, no obstante la estabilidad química, mecánica y térmica son superiores. La disminución de la conductividad protónica para el sistema HMS-PWA puede deberse por una parte a la incorporación del precursor híbrido. El MPS mejora la estabilidad mecánica porque genera redes de óxido de polietileno con cadenas largas y flexibles. Por otro lado, aumenta el número de enlaces entre la red orgánica e inorgánica, aumentando el grado de entrecruzamiento y mejorando la estabilidad química, sobre todo frente a la sulfonación. La disminución de la conductividad protónica podría deberse al aumento del grado de entrecruzamiento, lo que limitaría los caminos de conducción de los protones a través de la membrana.

\section{CONCLUSIONES}

Se han sintetizado membranas orgánico-inorgánicas vía polimerización orgánica y reacción sol-gel usando 3-metacriloxipropiltrimetoxisilano, estireno y 2hidroxietilmetacrilato (HMS) incorporando ácido fosfowolfrámico (PWA) y un proceso de sulfonación de los anillos aromáticos para dotar a las membranas híbridas con conductividad protónica. Las curvas de ATG-ATD muestran una excelente estabilidad térmica hasta $250^{\circ} \mathrm{C}$, indicando que estas membranas híbridas podrían utilizarse en PEMFC hasta esta temperatura. La estabilidad química y estructural de las membranas híbridas frente al proceso de sulfonación se ha confirmado por espectroscopia infrarroja. Las membranas presentan capacidades de intercambio iónico de hasta 0.43 $\mathrm{mmol} / \mathrm{g}$ y una absorción de agua del $10.3 \%$ para la membrana 
HMS-PWA sulfonada con $\mathrm{HSO}_{3} \mathrm{Cl}$, manteniendo buenas propiedades químicas y mecánicas. La conductividad aumenta con la temperatura, la humedad relativa y la concentración de los grupos $\mathrm{SO}_{3} \mathrm{H}$, llegándose a alcanzar valores de $3.210^{-3}$ $\mathrm{S} / \mathrm{cm}$ a $130^{\circ} \mathrm{C}$.

\section{AGRADECIMIENTOS}

Los autores agradecen al Ministerio de Educación y Ciencia la concesión de una beca FPI y un contrato Ramón y Cajal. Este trabajo ha sido financiado por el proyecto MAT2006-4375 y desarrollado en el marco de la Red nacional de Pilas de Combustible, Baterías avanzadas e Hidrógeno CSIC-Universidad. Los autores reconocen la ayuda aportada por Laura Peláez y Luis Contreras en el desarrollo del trabajo experimental.

\section{REFERENCIAS}

1. S. J. Padisson, Proton conduction mechanisms at low degrees of hydration in sulfonic acid-based polymer electrolyte membranes, Annu. Rev. Mater. Res., 33, 289-319 (2003).

2. K. Kordesch, G. Simader, Fuel cells and their applications, VCH, Weinheim (1996).

3. G. Alberti, M Casciola, Composite membranes for medium-temperature PEM fuel cells, Annu. Rev. Mater. Res., 33, 129-154, (2003)

4. K.D. Kreuer, On the development of proton conducting materials for technological applications, Solid State Ion., 97, 1-15, (1997).

5. M. Ciureanu, H. Wang, Electrochemical Impedance Study of ElectrodeMembrane Assemblies in PEM Fuel Cells: I. Electro-oxidation of $\mathrm{H}_{2}$ and $\mathrm{H}_{2} / \mathrm{CO}$ Mixtures on Pt-Based Gas-Diffusion Electrodes, J. Electrochem. Soc., 146, 4031-4040, (1999).

6. J. Rozière, J.J. Deborah, $<<$ Non-fluorinated polymer materials for proton exchange membrane fuel cells $>>$, Annu. Rev. Mater. Res., 33, 503-555, (2003).

7. L. C. Klein, M. Aparicio, F. Damay. Handbook of Sol-Gel Science and Technology, Processing, Characterization and Applications, volume III: Applications of Sol-Gel Technology, pp. 311-328, Kluwer Academic Publishers 2004.

8. I. Honma, J-D. Kim, Proton conducting polymethylsiloxane/metal oxide membranes added with phosphotungstic acid (II), Electrochim. Acta, 49, 3429-3433, (2004).

9. M. Aparicio, J. Mosa, M. Etienne, A. Durán, Proton-conducting methacrylatesilica sol-gel membranes containing tungstophosphoric acid, J. Power Sources, 145, 231-236, (2005).
10. C. Sánchez, P. Gómez- Romero, Functional hybrid materials, Wiley-VCH, 2004.

11. M. Aparicio, J. Mosa, A. Durán, Hybrid organic-inorganic nanostructured membranes for high temperature Proton Exchange Membranes Fuel Cells (PEMFC) , J of Sol-Gel Sci. and Tech., 40, 309-315, (2006).

12. U. Lavrencic Stangar, N. Groselj, B. Orel, A. Schmitz, Ph. Colomban, Proton-conducting sol-gel hybrids containing heteropoly acids, Solid State Ion., 145, 109-118, (2001).

13. P. Staiti, S Freni, S. Hocevar, Synthesis and characterization of protonconducting materials containing dodecatungstophosphoric and dodecatungstosilic acid supported on silica, J. Power Sources, 79, 250-255, (1999).

14. Stangar UL, Groseli N, Orel B, Schmi1z A, Colomban P, Proton-conducting sol-gel hybrids containing heteropoly acids, Solid State Ion., 145, 109-118, (2001).

15. Stangar UL, Orel B, Yince J, Jovanovski Y, Spreizer H, Yuk AS, Hocevar $S$, Silicotungstic acid/organically modified silane proton-conducting membranes, J.Solid State Electrochem., 9, 106-113, (2005)

16. A. M. Herring, Inorganic-polymer composite membranes for proton exchange membrane fuel cells, Polymer reviews, 46, 245-296 (2006)

17. U. Mioc, M. Davidovic, N. Tjapkin, A. Novak, Equilibrium of the protonic species in hydrates of some heteropolyacids at elevated temperatures, Solid State Ion., 46, 103-109, (1991)

18. I. Honma, H. Nakajima, S. Nomura, High temperature proton conducting hybrid polymer electrolyte membranes, Solid State Ion.,154-155, 707-712, (2002).

19. H. Nakajimaa, S. Nomura, T. Sugimoto, S. Nishikawa, I. Honma, J. Electrochem. Soc., Amphiphilic Organic/Inorganic Nanohybrid Macromolecules for Intermediate-Temperature Proton Conducting Electrolyte Membranes, 149, A1389-A1392, (2002).

20. A. S. Aricó, P. Creti, P.L. Antonucci, V. Antonucci, Comparison of Ethanol and Methanol Oxidation in a Liquid-Feed Solid Polymer Electrolyte Fuel Cell at High Temperature, Electrochem. Solid-State Lett., 1, 66-68, 1998.

21. P. Genova-Dimitrova, B. Baradie, D. Foscallo, C. Poinsignon and J. Y. Sánchez, Ionomeric membranes for proton exchange membrane fuel cell (PEMFC): Sulfonated polysulfone associated with phosphatoantimonic acid, J. Membr. Sci., 185, 59-71, (2001).

22. M. Aparicio, E. Lecoq, Y. Castro, A. Durán, Proton Conducting Organic/ Inorganic Sol-Gel Membranes Produced from Phenyltriethoxysilane and 3-Methacryloxypropyl Trimethoxysilane, J. Sol-Gel Sci. Tech., 34, 233-239, (2005).

23. M. Aparicio, Y. Castro, A. Durán, Synthesis and characterisation of proton conducting styrene-co-methacrylate-silica sol-gel membranes containing tungstophosforic acid, Solid State Ion., 176, 333-340, (2005).

24. M. Aparicio, J. Mosa, F. Sánchez, A. Durán, Synthesis and characterization of proton-conducting sol gel membranes produced from 1,4-bis (triethoxy silyl) benzene and (3-glycidoxypropyl) trimethoxysilane, J. Power Sources, 151, 57-62, (2005).

25. S. Slade, S. A. Campbell, T. R. Ralph, F. C. Walsh, Ionic conductivity of an extruded Nafion 1100 EW series of membranes, J. Electrochem. Soc., 149 (12), A1556-A1564, (2002). 